This paper is a postprint of a paper submitted to and accepted for publication in Electronics Letters and is subject to Institution of Engineering and Technology Copyright. The copy of record is available at IET Digital Library

\title{
Analysis and Mitigation of Antenna Effects on the Wideband Wireless Channel
}

Vit Sipal, Ben Allen, David Edwards

Affiliation: Dept. of Engineering Science, University of Oxford, Parks Road, Oxford OX1 3PJ, United Kingdom

This letter provides an alternative physical interpretation of the wireless channel impulse response using the knowledge of the antenna impulse responses. As a result, it can be shown that the clusters in the popular Saleh-Valenzuela model actually represent one multipath component only, not several rays as is often assumed. This knowledge has a significant impact on receiver architecture since the number of fingers in a rake receiver can be reduced significantly, for some systems by a factor of up to 8 . The drawback of the method is an increase in processing complexity in each finger, but it is anticipated that the overall processing complexity in receiver will be reduced.

Introduction: One of big advantage of ultrawideband (UWB) systems is the ability to resolve many multipath components (MPCs), therefore the system can receive energy carried by each MPC individually. Probably the most popular model describing an unltrawideband channel impulse response (IR) is the Saleh-Valenzuela model (SV-model) [1]. An example of a channel impulse response as described by this model is presented in Fig. 1. According to the model, the MPCs arrive in clusters. These clusters are depicted by the dotted line. Each cluster consists of several MPCs as it is assumed that due to the large bandwidth, these MPCs can be resolved in an UWB channel. The energy within a cluster of MPCs is assumed to decay exponentially over time, as does the energy of the clusters. The delays between the 
clusters and the individual MPCs within the clusters are, according to the SV-model, both random variables given by Poisson-distribution.

For a system designer, the optimal receiver that would capture the entire available energy would be a rake receiver with a finger for each resolvable MPC. Unfortunately, the number of MPCs predicted by the SV-model for standard ultrawideband environments is so high that such an optimal rake receiver architecture is far too complex and thus is not viable for real applications.

New interpretation of channel impulse response - theory: The SV-model in [1] was developed based on a number of channel measurement campaigns in order to provide a simple tool for modelling. In this work we postulate that the basic assumption of SV-model relating to the clustering of rays is not correct. We demonstrate that each cluster in the SVmodel represents only one MPC. In other words, the individual non-zero IR values within the cluster do not represent individual MPCs, and are shown in this letter to all belong to only one MPC.

The reason why the MPC is represented by a number of non-zero values rather than one Dirac-impulse, as is normally assumed, is due the distortion introduced by the antennas. Antennas have an impulse response, sometimes called the effective height [2], that relates the shape of voltage/current input pulse to the time domain waveform of the electric field around the antenna. If these impulse responses of the transmitter and receiver antennas are convolved with the pure channel impulse response (antenna effects excluded), impulse responses similar to Fig. 1 are obtained.

Additionally, the temporal proximity of rays within a cluster (as suggested by standard IEEE model [1]) represents spatial path differences of about $10-15 \mathrm{~cm}$. This may well be a reasonable assumption for later clusters, but there seems to be no physical reason for this within the first Line-of-sight component. This would suggest the presence of obstacles close to the Line-of-sight which indicates a partial NLOS measurement. 
Validation: To validate the postulation, the impulse response of an ideal channel in free space with no reflected paths was simulated and measured. The simulated link used discone antennas that are relatively popular in channel sounding measurements.

For the simulation over the frequency range of $0.1-25 \mathrm{GHz}$, the transmitter antenna impulse response was calculated using CST Microstripes ${ }^{\mathrm{TM}}$ together with the algorithm introduced in [3]. The receiver antenna impulse response was calculated from the impulse response of transmitter antenna according to relationships derived in [2]. Finally, the simulated channel impulse response was calculated as the convolution of these impulse responses considering all polarisations of these impulse responses as outlined in [2].

The measurement was conducted in an anechoic chamber for a TX-RX distance of $70 \mathrm{~cm}$ with a measurement bandwidth of $50 \mathrm{MHz}-30 \mathrm{GHz}$ and using the same antennas as those modelled in the simulation. The results of the simulation and measurement are presented in Fig. 2 which shows the normalized square of channel impulse response for each case.

From Fig. 2, firstly, a good agreement of theory and measurement is observed. The small discrepancy can be attributed to simulation errors and non-ideal measurement conditions. Secondly, even though there is only one ray present in the measurement and simulation, the channel impulse response does not resemble a single Dirac impulse. From this point of view, the distortion can be seen to be due to the antenna impulse responses. Therefore, individual rays within a cluster that were believed to stem from several multipath components are actually the manifestation of the antenna impulse response.

We believe that the reason, why the individual non-zero values within a cluster are often attributed to individual MPCs, arises from the insufficient bandwidth of channel measurements (often less than $3 \mathrm{GHz}[1]$ ) and because the concept of antenna impulse response is not yet used as a standard antenna descriptor. The impact is, however, negligible for signal bandwidths below $1 \mathrm{GHz}$ as for these signals the impulse response duration is negligible compared to the length of a symbol. 
Additionally, the assumption that rays within a cluster originate from the antenna impulse response can also explain the differences among measurements carried out by different research groups simply because they used different antennas. To illustrate this, the impulse response of the channel with one MPC only was modelled for three different discone antennas, each of same shape but scaled in size. The results are plotted in Fig. 3, and show that for the same measurement the internal cluster characteristics (especially the decay) of the SV model varies significantly whilst the inter-cluster characteristics (i.e. decay and cluster arrival rate) would remain unchanged. The change is caused by different lower cut off frequencies of the antennas and from different spatial distribution of currents that is due to different antenna dimensions.

Practical interpretation: The consequence of this investigation for the design of UWB systems is significant. Since "rays" within a cluster are not random variables, but are determined by the type of the antennas and their mutual orientation. Consequently in an optimal rake receiver with, several fingers assigned to rays from one cluster can be replaced by one finger using a correlation with the known deterministic impulse response of the antenna. This is illustrated by Fig 4. where the impulse response of the link from Fig. 2 is sampled at the rate corresponding to $167 \mathrm{ps}$ which is the rate used in [1]. As can be seen, under the assumption that each non-zero value stems from an individual ray, one can observe that 8 of the sampled points fall into the energy interval of $10 \mathrm{~dB}$ below the impulse response maximum value. This level is also plotted in Fig. 4. Practically, a rake receiver processing these rays would have an individual finger for each of these 8 points. Obviously, this number would be higher for sampling rates above 167 ps or lower for an antenna pair with shorter impulse response.

Thus the practical benefit is, that if several non-zero values of IR are taken as part of one MPC, the number of fingers would be reduced by a factor as high as 8 as suggested by the experimental results. Moreover, due to the use of the correlation operation with a 
deterministic impulse response it can be expected that all the "rays from one cluster", actually belong to one MPC, and will be summed.

However, it should be noted that the complexity of each finger would consequently increase. This is because the impulse response of each MPC component is given by the type of antennas employed, their mutual position and the angle of arrival of each MPC. Such processing must be included into each finger as each MPC has its individual angle of arrival and therefore represents the mutual position of antennas. A perfect process using the exact antenna impulse response for each MPC would impose a significant challenge.

For practical situations, however, a simplified process can be applied. Firstly, the spatial variation of antenna impulse response in terms of waveform distortion is not large as can be seen in antenna correlation pattern descriptors reported in [4]. Additionally in terms of their impulse responses, antennas act as differentiators, and the impulse responses in Fig. 3 appear as the convolution of two non-ideal differentiators. Thus, it may be possible to formulate a simple parametric model of waveform shape for antenna impulse responses that would simplify the problem of determining the exact impulse response for a given antenna position in space.

Conclusion: In this letter, the standard interpretation of the wideband wireless channel impulse response has been challenged. It is believed that the widely accepted explanation neglects the effect of antenna impulse responses. An alternative interpretation of the channel impulse response is provided and supported by both simulation and measurements. It is shown that with this new explanation, rays from one cluster in the SV-model actually belong to a single MPC. The practical importance is that it allows a significant reduction of the number of fingers in rake receivers in exchange for a slight increase of finger complexity. It is expected that with a simplified processing regime, the total complexity of a receiver will be reduced. 


\section{References:}

[1] Molisch, A.F.; Foerster, J.R.; Pendergrass, M.; "Channel models for ultrawideband personal area networks," Wireless Communications, IEEE Transactions on , vol.10, no.6, pp. 14- 21, Dec. 2003

[2] Shlivinski, A.; Heyman, E.; Kastner, R.; "Antenna characterization in the time domain," Antennas and Propagation, IEEE Transactions on , vol.45, no.7, pp.1140-1149, Jul 1997

[3] Sipal, V.; Allen, B.; Edwards, D.; "Modelling of antenna pattern descriptors for antenna performance evaluation," Modelling and Simulation, 2010. AMSE '10. International Conference on, June 2010 (accepted)

[4] Zhang, X.; Lu, W.; Zeng, Y.; "Pattern descriptors for baseband-pulse-antenna," Electromagnetic Compatibility and 19th International Zurich Symposium on Electromagnetic Compatibility, 2008. APEMC 2008. Asia-Pacific Symposium on, vol., no., pp.331-334, 19-23 May 2008

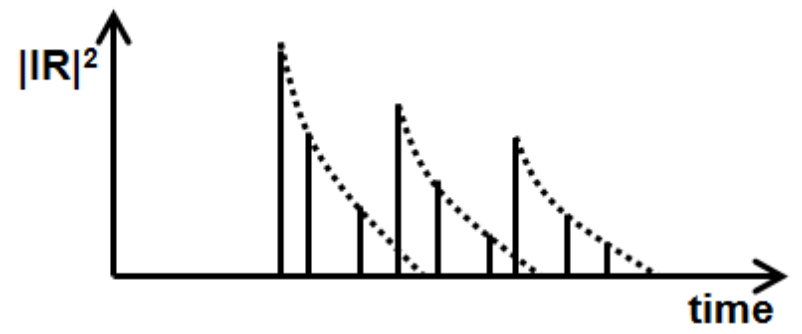

Fig. 1 Sample channel impulse response according to the SV model

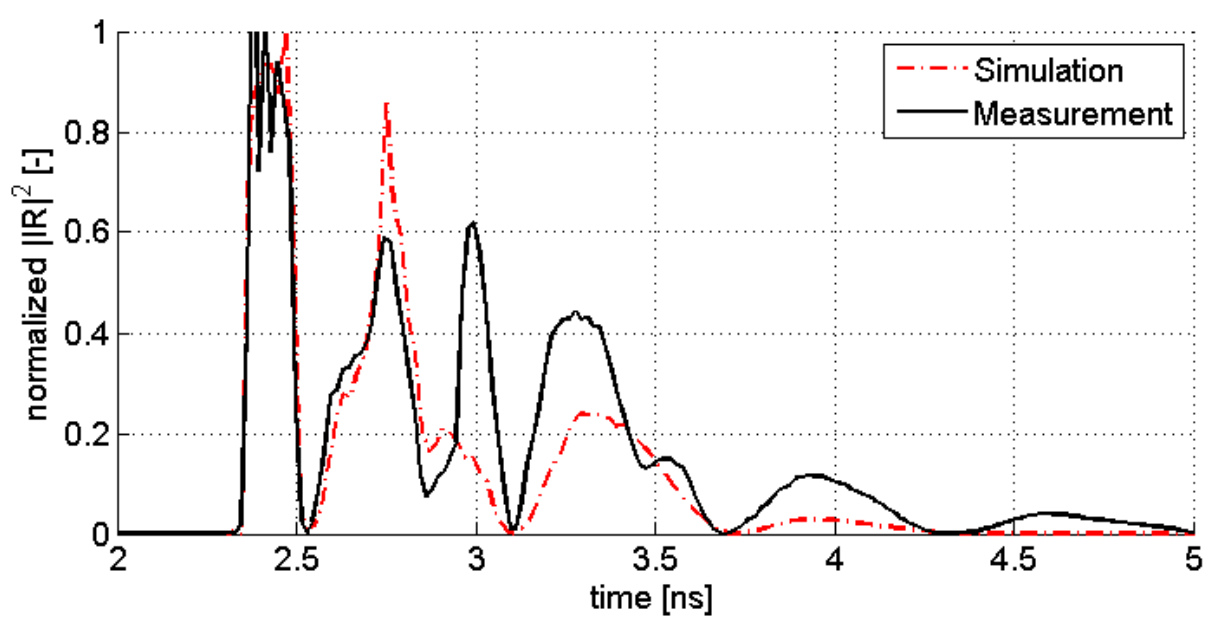

Fig. 2 Simulated and measured impulse response of a single path channel 


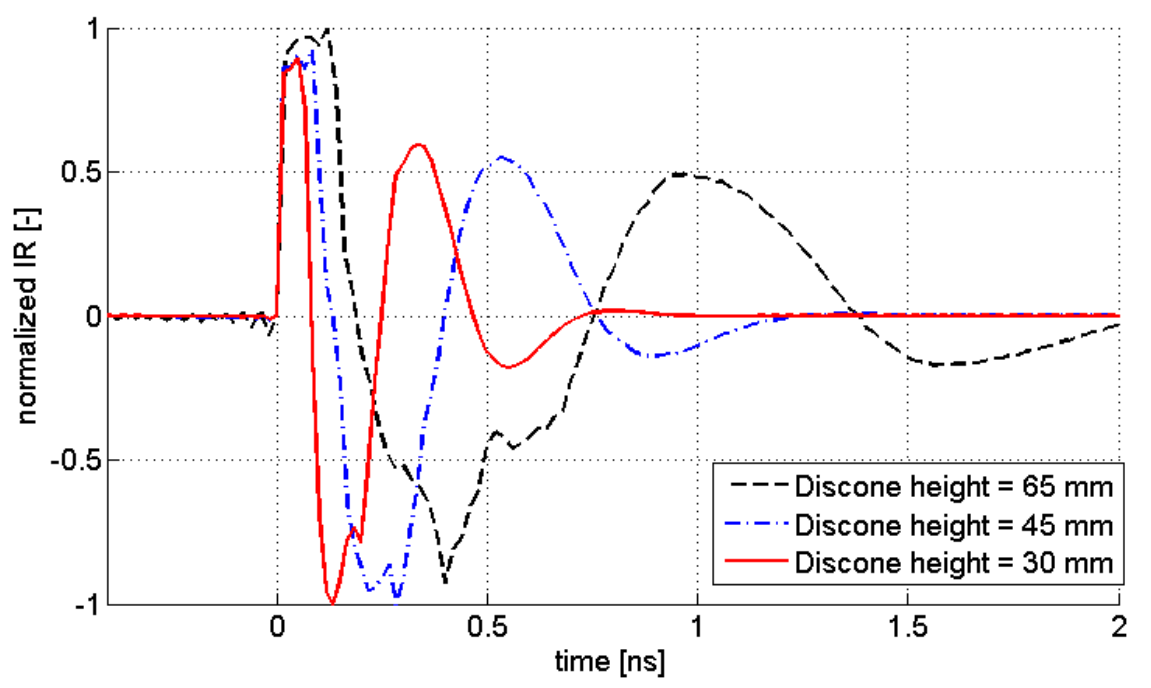

Fig. 3 Variation of channel impulse response with antenna size

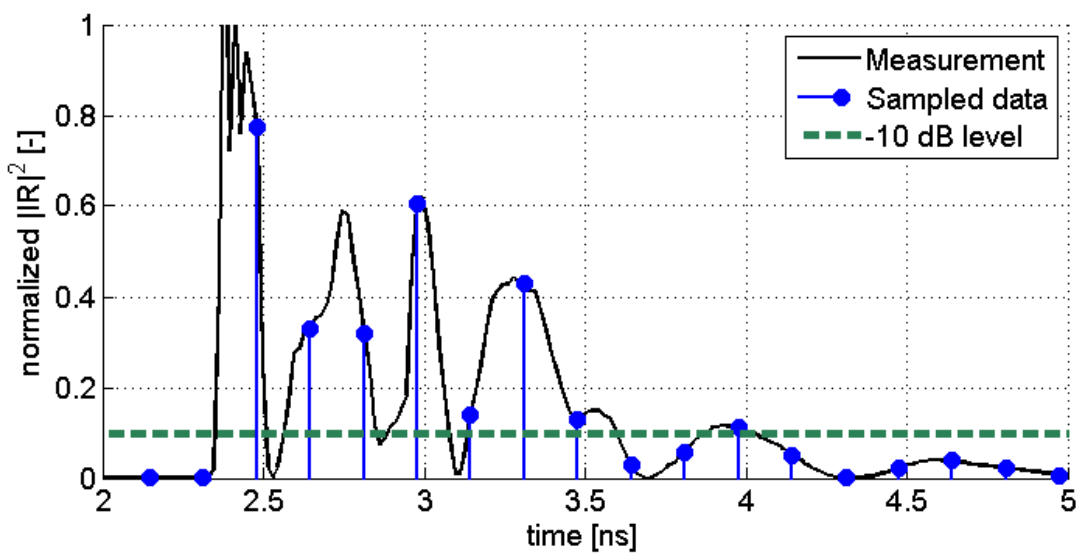

Fig. 4 Measured channel IR sampled at 167 ps rate 